\title{
DC polarization electric field, current density, and plasma density measurements in the daytime equatorial electrojet
}

\author{
Robert F. Pfaff, Jr., Mario H. Acuña, and Paul A. Marionni \\ NASA/Goddard Space Flight Center, Greenbelt, MD
}

\author{
Nalin B. Trivedi \\ Instituto Nacional de Pesquisas Espacias, São José dos Campos, Brazil
}

\begin{abstract}
Measurements of the vector DC electric field, current density, and plasma number density were gathered in the daytime equatorial electrojet on a sounding rocket launched from Alcântara, Brazil. The data set provides a self-consistent picture of the electrodynamics of the daytime electrojet, permitting a detailed comparison of the altitude profiles of the vertical component of the DC electric field, $\mathrm{E}_{\mathrm{ver}}$, the current density, $\mathrm{J}$, and the plasma number density, $\mathrm{N}_{\mathrm{e}}$. Good agreement in the upper electrojet region between both the magnitude and the profile of the plasma drifts, calculated independently from $E_{\text {ver }} / B$ and $\mathrm{J}_{\mathrm{zona}} / \mathrm{qN}_{\mathrm{e}}$, demonstrates that the equatorial electrojet is a Hall current consisting of electron motion driven by a vertical DC electric field. The data support the basic Cowling conductivity ideas of electron flow at the dip equator within a narrow conducting layer in which the ions are at rest.
\end{abstract}

\section{Overview, Launch Conditions, and Instruments}

In the Guará Campaign, a series of NASA rockets were launched to study the electrodynamics of the equatorial electrojet [see Pfaff et al., this issue]. Excellent data were returned from three rocket experiments which each revealed vertical DC electric fields that peak near $105 \mathrm{~km}$ within the electrojet, coincident with where the observed current density is also maximum. In this paper, we present results from one of these rockets with the aim of providing, insofar as possible, a complete electrodynamic picture of the daytime equatorial electrojet based on a "snapshot" of data gathered by the rocket-borne probes.

On September 21, 1994, the ground-based magnetometer at Alcântara, Brazil revealed a strong positive deflection in the $\Delta \mathrm{H}$ component, as shown in Figure 1. The data show variations with periods of about an hour and amplitudes of roughly $\pm 15 \mathrm{nT}$ about a mean peak excursion of about $100 \mathrm{nT}$. Magnetometer data from an off-equator station (Vassouras, mag. lat. $\sim 20^{\circ}$ ) also showed a large $\Delta H$ excursion of approximately 60-70 nT, implying that an extended $\mathrm{Sq}$ current system was present. Coincident with these observations, the CUPRI radar observed strong VHF backscatter echoes throughout the morning in the lower electrojet, $95-105 \mathrm{~km}$ [Swartz, personal communication, 1994]. No high altitude (106-110 km) echoes associated with primary two-stream wave excitations were observed.

With these electrojet conditions evidenced in the ground-based observations, NASA rocket 21.111 was launched at 10:56:00 L.T. (13:56:00 U.T.) from Alcântara, Brazil, along an azimuth in the magnetic east direction. The payload achieved an apogee of

\section{Copyright 1997 by the American Geophysical Union.}

Paper number 97GL01536.

0094-8534/97/97GL-01536\$05.00
$120.2 \mathrm{~km}$ approximately 3 minutes later, and returned good data from all of its instruments. At the time of apogee, the ground magnetometer $\Delta \mathrm{H}$ deflection was $105 \mathrm{nT}$.

The rocket payload was equipped with double probes of different lengths to measure the DC and wave electric fields, a high precision, three-axis flux-gate magnetometer to determine the current density, and a plasma frequency probe to measure the electron density. A set of spaced receivers was included to measure the wavelength and phase velocity of the plasma waves.

After exiting the lower atmosphere, the attitude control system oriented the spin axis to within 1 degree of the magnetic field direction. As shown in Figure 2, the spin plane, where the double probes gather their most reliable measurements, was thus perpendicular to the magnetic field and co-planar with the plane containing the electric fields [see Pfaff, 1991]. A gyroscope provided attitude data with an accuracy of 1 degree.

\section{Measurements}

DC Electric Field. Electric field data from two perpendicular double probes gathered near apogee $(120 \mathrm{~km})$ are shown in the spacecraft frame in Figure 3. The large sine waves occur at the spin period of roughly $2 \mathrm{~s}$. Gathered with $4.4 \mathrm{~cm}$ diameter spherical sensors separated along $5.5 \mathrm{~m}$ and $3.0 \mathrm{~m}$ baselines, these fields represent the measured potentials divided by their respective boomlengths. The electric field magnitudes are the same in the two panels indicating that the potentials scale linearly with boomlength and that valid fields have been measured. Notice that the two components differ in phase by $90^{\circ}$ as

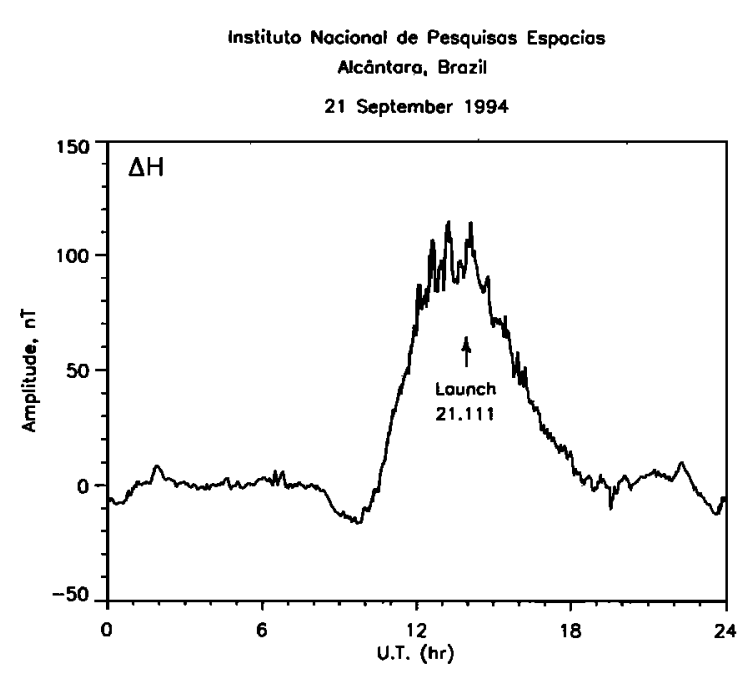

Figure 1. INPE magnetometer $\Delta H$ deflection at Alcântara, Brazil on September 21, 1994. Local Time = U.T. -3 hours. 


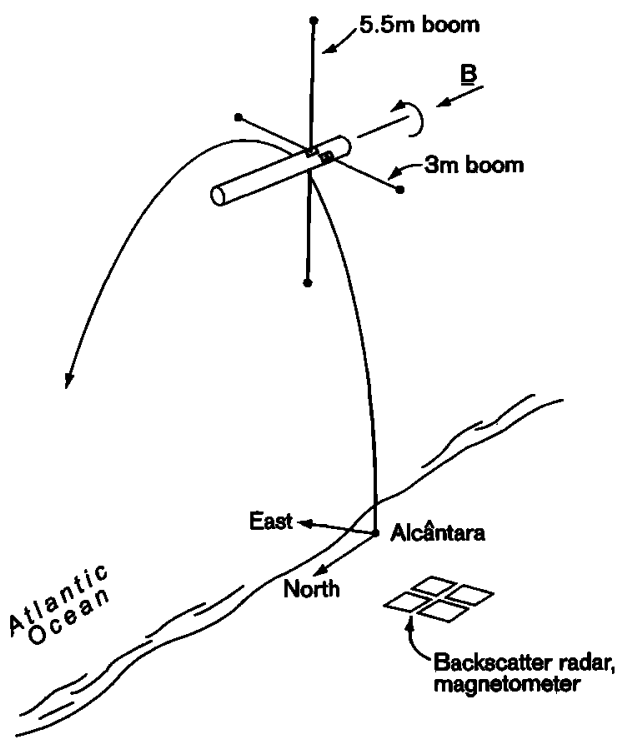

Figure 2. Payload orientation with the spin plane, which contain the double probes, perpendicular to the magnetic field direction.

expected from perpendicular, spinning probes. The data also depict small "sun notches" twice per spin, which are due to photoelectron current asymmetries which occur when one sphere is in the shadow of the payload and the other is in sunlight.

The sun notches also provide independent attitude information. Since this rocket was launched on the equinox, a few degrees from the geographic equator, and near local noon, the sun was nearly overhead during the flight and hence the notches occur near to that part of the spin cycle when each boom pair rotated through the vertical direction. Since the ambient and $\mathbf{V} \times \mathbf{B}$ electric fields (due to the horizontal motion of the payload, discussed below) were almost entirely in the vertical direction when the payload was near apogee, the sun notches appear near the extrema of the spin modulated electric fields in Figure 3.

The largest contribution to the electric field measurements on a spacecraft moving through the equatorial ionosphere is that due to the $\mathbf{V} \times \mathbf{B}$ fields created by the motion of the payload across the Earth's ambient magnetic field, where $\mathbf{V}$ is the rocket velocity in the earth-fixed frame and $\mathbf{B}$ is the ambient magnetic field. The contributions due to $\mathbf{V} \times \mathbf{B}$ are shown by the dotted lines in Figure 3 and represent the fields that would have been measured if no ambient (i.e., geophysical) electric fields were present. Our next step is to subtract these $\mathbf{V} \times \mathbf{B}$ fields from the measurements and rotate the resulting components from the spacecraft frame into a more meaningful coordinate system in order to better interpret the results. Based on measurements and theory, we make the assumption that $\mathbf{E} \cdot \mathbf{B}=\mathbf{0}$ for the DC electric fields that concern us here. After these calculations are carried out, the fields are rotated into geomagnetic coordinates and the results for the downleg are plotted vs. altitude in Figure $4(a, b)$.

Notice immediately in the electric field data in Figure 4 that there is a large DC electric field in the vertical direction and that this peaks near $105 \mathrm{~km}$ with a value of approximately $10 \mathrm{mV} / \mathrm{m}$. This is the polarization electric field that drives the electrojet current. The value of the electric field is about $2.5 \mathrm{mV} / \mathrm{m}$ at apogee which is consistent with satellite measurements at higher altitudes of the Sq dynamo electric field for this local time [Maynard et al., 1988]. The electric field in the lower electrojet region (90-104 km) is also non-zero and represents vertically oriented potential differences sustained in this region. Unfortunately, the lower altitude electric field data were contaminated with wake effects, so it is difficult to interpret the data in this altitude region, as we discuss further on below.

The zonal component, $\mathbf{E}_{\text {zonal }}$, is approximately $1 \mathrm{mV} / \mathrm{m}$ eastward at apogee. Although the direction of this component agrees with that of the expected, daytime zonal Sq electric field, its value is larger than average values of $0.3 \mathrm{mV} / \mathrm{m}$ inferred from incoherent scatter measurements at higher altitudes [Fejer et al., 1979]. It is also somewhat larger than the daytime zonal electric fields deduced from phase velocity measurements of backscatter echoes near $150 \mathrm{~km}$ [Kudeki and Fawcett, 1993]. Average measurements of the vertical ion plasma drift (corresponding to the zonal electric field) gathered by satellite probes at this local time [Coley and Heelis, 1989] correspond more closely to the zonal electric field value that we report here. In general, despite possible errors in the measurement (see discussion below), in contrast to the vertical component, the measured zonal electric field component in the daytime electrojet is small and eastward above $104 \mathrm{~km}$. Larger variations are observed in the lower electrojet created by gradient drift-driven perturbations.

Errors in the electric field measurement vary with altitude as they are a strong function of both the ambient plasma and neutral number densities. The largest source of error is wake effects which occur as each sphere rotates through the trailing velocity cone of the payload once per spin. Such effects are significant since the payload was aligned in the north-south direction and the rocket was launched along an eastward trajectory. In other words, the same payload orientation (Figure 2) which enables the zonal and vertical electric fields to both be determined within the spin plane also enhances the wake effects that would otherwise be minimized for a vertically oriented payload. These wakeinduced perturbations are worse for shorter boom systems and could be alleviated in future missions with longer booms.

At this stage in the analysis, we estimate that the errors associated with the DC electric fields shown in Figure 4(a,b) are $\pm 0.5 \mathrm{mV} / \mathrm{m}$ above $105 \mathrm{~km}$, increasing to values possibly as large as $\pm 3 \mathrm{mV} / \mathrm{m}$ at $100 \mathrm{~km}$. In this presentation, we have removed the sun notches discussed above. The data have also been smoothed using averaging and detrending techniques to reveal the slowly varying component of the electric field components.

Current density. A high precision, three-axis flux-gate magnetometer was built specifically for this experiment and was extended at the end of a $1.3 \mathrm{~m}$ rigid, fiberglass boom. The data were digitized on-board using a 16-bit analog-to-digital converter for which the least significant bit corresponded to $\pm 1.5 \mathrm{nT}$. The payload was designed to minimize spurious magnetic signals and
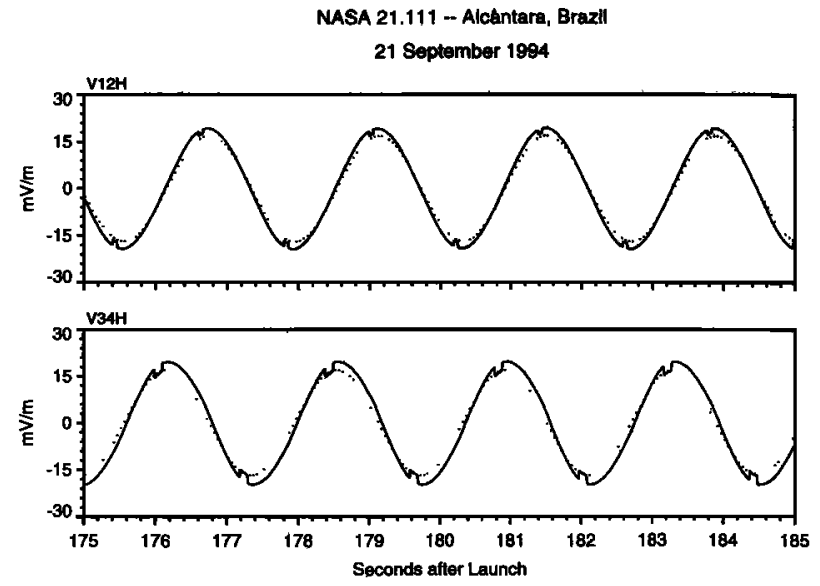

Figure 3. Examples of raw data from the $5.5 \mathrm{~m}$ and $3.0 \mathrm{~m}$ electric field detectors from the apogee region (near $120 \mathrm{~km}$ ). 


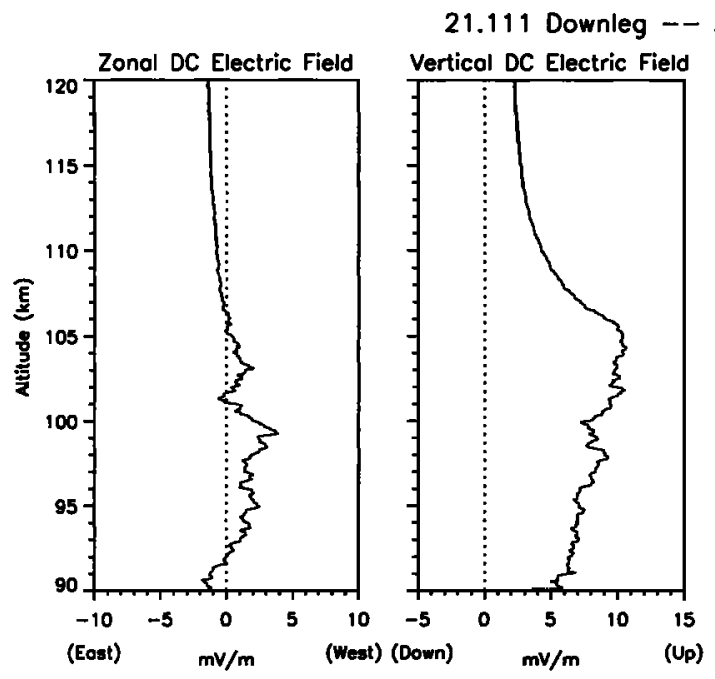

(o)

(b)

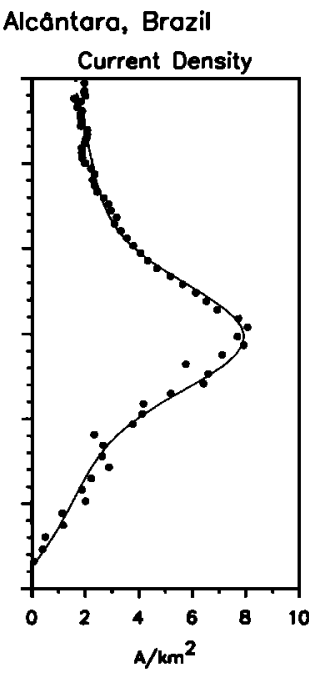

(c)

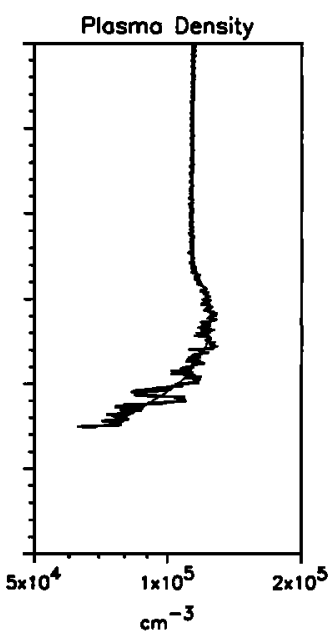

(d)

Figure 4. Zonal (a) and vertical (b) electric field profiles for the downleg. Current density (c) determined from the vertical gradient in the measured magnetic field data. Plasma number density (d) determined from the plasma frequency probe. The thin solid lines in (c) and (d) represent empirical fits to these data.

was calibrated in the NASA/Goddard Space Flight Center Magnetic Test Facility. Using Ampère's law, $\nabla \times \mathbf{B}=\mu_{\mathrm{o}} \mathbf{J}$, and assuming no vertical currents, the derivative of the magnitude of the magnetic field with respect to altitude is determined. (Here, $\mathbf{J}$ is the current density and $\mu_{o}$ is the permeability of free space.) The computed current density consists of the magnetic zonal component, $\mathrm{J}_{\text {zonal, }}$, and is plotted in Figure 4(c).

The current density profile peaks at $8 \mathrm{~A} / \mathrm{km}^{2}$ near $105 \mathrm{~km}$. This altitude is slightly lower than that corresponding to the peaks of the current density profiles observed off the coast of Peru [e.g., Maynard, 1967; Richmond, 1973] and India [e.g., Maynard and Cahill, 1965; Sampath and Sastry, 1979]. The magnitude of the current density is consistent with the ground $\Delta H$ magnetometer deflection of $105 \mathrm{nT}$, as shown by Richmond [1973] and Sampath and Sastry [1979] for similar data sets.

Plasma Density. The plasma number density was measured using a plasma frequency probe which locks onto a parallel resonance corresponding to the upper hybrid frequency [Jensen and Baker, 1992]. Since the magnetic field is known, the electron gyro frequency is easily determined, yielding the plasma frequency from which the electron number density is obtained. The data are shown in Figure 4(d) and reveal a peak near $104 \mathrm{~km}$ of $1.3 \times 10^{5} \mathrm{~cm}^{-3}$, which is consistent with other daytime equatorial observations and with simultaneous measurements from a nearby ionosonde in São Luis (Abdu, personal communication, 1994). The variations below $105 \mathrm{~km}$ are due to the gradient-drift driven $\mathrm{km}$-scale structures. The small negative gradient of the plasma density between 104 and $107 \mathrm{~km}$ is a real effect and has been noted in other data sets [e.g., Pfaff et al., 1987]. The instrument lost "lock" at approximately $97 \mathrm{~km}$ on the downleg. Data (not shown) from a Langmuir probe corroborate these measurements and extend this profile to lower altitudes.

\section{Discussion}

The simultaneous measurements of the DC electric field, current density, and plasma number density permit a detailed investigation of the electrodynamics of the daytime equatorial electrojet and an opportunity to test the long held Cowling conductivity theory concerning the electrojet formation. Notice immediately in Figure 4 that the vertical electric field peaks where the electrojet current is maximum at $105 \mathrm{~km}$. Since the current density is a function of the plasma number density, a more relevant comparison is of the electron drift velocity associated with each profile.

Drift Velocity Comparisons. The drift velocity, $V_{d}$, of a plasma population of equal number of electrons and ions in thermal equilibrium is given by:

$$
\mathbf{V}_{\mathrm{d}}=\mathrm{V}_{\mathrm{i}}-\mathrm{V}_{\mathrm{e}}=\mathrm{J} / \mathrm{qN} \mathrm{N}_{\mathrm{e}}
$$

in which $V_{i}$ and $V_{e}$ are the ion and electron drift velocities and $q$ is the electronic charge. This profile has been calculated as a function of altitude in which we have fit empirical smooth functions to the current density and plasma number density represented by the thin lines in Figures 4(c) and 4(d). The results are shown by the solid line in Figure 5 and show a peak velocity

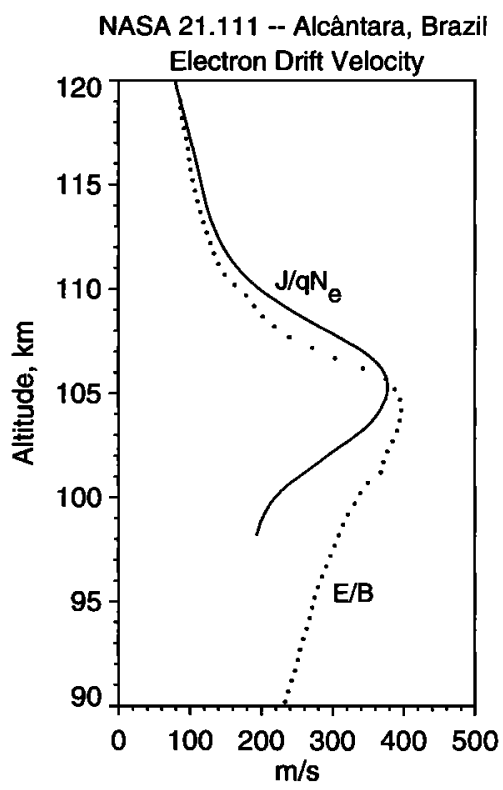

Figure 5. Comparisons of the drift velocity using the current density and plasma number density (solid line) and that from the $\mathrm{E}_{\text {vert }} / \mathrm{B}$ measurements (dotted line). 
of $370 \mathrm{~m} / \mathrm{s}$ at $105 \mathrm{~km}$. The drift velocity can also be independently calculated from $\mathbf{E} \times \mathbf{B}$ motion using the electric field measurements. Assuming that the ions are at rest (i.e., $\mathbf{V}_{\mathbf{i}}=$ 0 ) and ignoring the small zonal electric field contribution, the horizontal electron drift is simply

$$
\mathrm{V}_{\mathrm{d}}=\mathrm{V}_{\mathrm{e}}=\mathrm{E}_{\text {vert }} / \mathrm{B}
$$

This profile is shown in Figure 5 by the dotted line, and peaks at $390 \mathrm{~m} / \mathrm{s}$ at roughly $104.5 \mathrm{~km}$. Since we have fit the electric field profile with a smooth function, the actual peak altitude may vary somewhat, particularly since the data is modulated by the $\mathrm{km}$ scale plasma instability electric field structures.

For the upper electrojet region (> $104 \mathrm{~km}$ ), notice not only the similarity of the profiles with altitude, but also that the two drift velocities peak with approximately the same magnitude. The good agreement of the two drift velocities support the view that the electric field is driving a Hall current of electrons in accordance with (1) and (2). The slight departures above the peak may be due to uncertainties in producing the smoothed profiles and from vertical currents ignored in computing the curl of $B$. In the lower electrojet $(<104 \mathrm{~km})$, the curves do not agree as well. Although it is not unreasonable for vertical electric fields to be sustained at lower altitudes at the equator, the exponential decrease in plasma density results in far fewer current carriers. Wake effects may have altered the electric field data in the lower electrojet. Such effects would not hamper the magnetic field measurements or the current density profile.

Finally, the measured plasma drift velocities are supported by the fact that neither the in-situ wave measurements (not shown) nor the CUPRI radar data (not shown) reveal any evidence for primary two-stream waves above $105 \mathrm{~km}$ in the region where such waves are known to be excited by strong electrojet currents. The electron drift threshold for this instability at $105 \mathrm{~km}$ at the equator is roughly 1.2 times the acoustic velocity [e.g., Fejer and Kelley, 1980]. Since this threshold is about $430 \mathrm{~m} / \mathrm{sec}$, the measured drift velocities are consistent with the lack of evidence of two-stream waves and the conclusion that the electron drift speed was below the two-stream instability threshold.

Cowling Conductivity. Ionospheric currents may be represented by $\mathrm{J}=\underline{\underline{\sigma}} \cdot \mathbf{E}$, where $\underline{\underline{\sigma}}$ is the conductivity tensor which is highly anisotropic due to the influence of the Earth's magnetic field. For cases where the magnetic field is strictly horizontal (i.e., dip angle $=0$ ) and vertical currents are assumed to be zero (because of the small, finite height of the conducting strip), it is straightforward to show [e.g., Kelley, 1989]:

and

$$
\mathrm{J}_{\text {zonal }}=\left(\sigma^{2}{ }_{\mathrm{H}} / \sigma_{\mathrm{P}}+\sigma_{\mathrm{P}}\right) \mathrm{E}_{\mathrm{zonal}}=\sigma_{\mathrm{C}} \mathrm{E}_{\mathrm{zonal}}
$$

$$
\mathrm{E}_{\mathrm{vert}}=-\left(\sigma_{\mathrm{H}} / \sigma_{\mathrm{P}}\right) \mathrm{E}_{\mathrm{zonal}}
$$

where $\sigma_{\mathrm{H}}$, and $\sigma_{\mathrm{P}}$ are the Hall and Pedersen conductivities and have their usual definitions [e.g., Kelley, 1989], and $\sigma_{C}$ is the Cowling conductivity. The Cowling conductivity may be viewed as an enhancement of the Pedersen conductivity.

Inserting an $\mathrm{E}_{\text {zonal }}$ value of $0.3 \mathrm{mV} / \mathrm{m}$ into (4) yields a polarization field of about $11 \mathrm{mV} / \mathrm{m}$ peaking near $100 \mathrm{~km}$. Thus, the measured ambient $E_{\text {zonal }}$ of about $\sim 1 \mathrm{mV} / \mathrm{m}$ at apogee would yield an unrealistically large $E_{\text {vert }}$. Although possibly explained by measurement uncertainties, it is also likely that quasi-global values of $\mathrm{E}_{\text {zonal }}$ should be used in (4) and not specific, local ones. The peak altitude of the vertical electric field predicted by (4) occurs about $5 \mathrm{~km}$ lower than the observed peak altitude of $\sim 105 \mathrm{~km}$, for reasonable collision frequencies [e.g., Forbes, 1981]. Similar discrepancies between current density peak altitudes and those predicted by (4) have been studied by several authors [e.g., Forbes, 1981; Stening, 1985]. The fact that the independently-derived electron drift profiles driven by the measured vertical electric field and the measured current density (Figure 5) both disagree with the altitude profile predicted by (4) further underscores the limitations of the simple theory.

A more complete analysis of the DC electric field, current density, and plasma number density must include neutral winds, vertical currents, and global electrodynamic considerations. Since the measured DC polarization electric field is consistent with the measured current and plasma number density and since the measured zonal and vertical electric field components follow the general response predicted by (4), we conclude that the Cowling conductivity at the dip equator is a viable explanation for the creation of the equatorial electrojet.

Acknowledgments. We acknowledge the expertise of the engineers and technicians of the NASA/Wallops Flight Facility who designed, built, tested, and flew the payload from which the data are presented here. In particular, we salute the payload manager, Mr. Bruce Scott.

\section{References}

Coley, W. R., and R. A. Heelis, "Low-Latitude zonal and vertical ion drifts seen by DE 2," L. Geophys. Res., 94, 6571, 1989.

Fejer, B G., and M. C. Kelley, "lonospheric irregularities," Rev. Geophys., 18, 401, 1980.

Fejer, B. G., et al., "Dependence of equatorial F region vertical drifts on season and solar cycle," J.Geophys. Res., 84, 5792, 1979.

Forbes, J. M., "The equatorial electrojet," Rev. Geophys, 19, 469, 1981.

Jensen, M. D., and K. D. Baker, "Measuring ionospheric electron density using the plasma frequency probe," J.Space. Rock., 29, 91, 1992.

Kelley, M. C., The Earth's Ionosphere, Academic Press, 1989.

Kudeki, E., and Fawcett, C., "High resolution observations of $150 \mathrm{~km}$ echoes at Jicamarca," Geophys. Res. Lett., 20, 1987, 1993.

Maynard, N. C., "Measurements of ionospheric currents off the coast of Peru," J. Geophys. Res., 72, 1863, 1967.

Maynard, N. C., and L. J. Cahill, "Measurement of the equatorial electrojet over India," J. Geophys. Res., 70, 5923, 1965.

Maynard, N. C., et al., "Average low-latitude meridional electric fields from DE 2 during solar maximum," J.Geophys. Res., 93, 4021, 1988.

Pfaff, R. F., "Rocket observations in the equatorial electrojet: Current status and critical problems," L.Atmos. Terr. Physics, 53, 709, 1991.

Pfaff, R. F., et al., "Electric field and plasma density measurements in the strongly-driven daytime equatorial electrojet: 1 . The unstable layer and gradient drift waves," J. Geophys. Res., 22, 13578, 1987.

Richmond, A. D., "Equatorial electrojet--I. Development of a model including winds and instabilities," J.Atmos. Terr. Phys., 35, 1083, 1973.

Sampath, S., and T. S. G. Sastry, "Results from in-situ measurements of ionospheric currents in the equatorial region--I," J. Geomag. Geoelectr., 31, 373, 1979.

Stening, R. J., "Modeling the equatorial electrojet," I. Geophys. Res., 90 , $1705,1985$.

M. H. Acuña, P. A. Marionni and R. F. Pfaff, Jr., NASA/Goddard Space Flight Center, Greenbelt, MD, 20771.

N. B. Trivedi, Instituto Nacional de Pesquisas Espacias, Cx. Postal 5I5, São José dos Campos, Brasil

(Received: November 20, 1996; accepted: March 10, 1997) 